\title{
Review Article \\ Systematic Review and Meta-Analysis of the Utility of Circular RNAs as Biomarkers of Hepatocellular Carcinoma
}

\author{
Qingqin Hao, ${ }^{1}$ Yadi Han, ${ }^{2}$ Wei Xia, ${ }^{1}$ Qinghui Wang, ${ }^{1}$ and Huizhong Qian ${ }^{1}$ \\ ${ }^{1}$ Department of Clinical Laboratory, Wuxi Red Cross Blood Center, Wuxi 214000, Jiangsu, China \\ ${ }^{2}$ Department of Clinical Laboratory, The Affiliated Cancer Hospital of Zhengzhou University and Henan Cancer Hospital, \\ Zhengzhou 450008, Henan, China
}

Correspondence should be addressed to Huizhong Qian; qwz5878797@163.com

Received 8 November 2018; Accepted 31 March 2019; Published 2 May 2019

Academic Editor: Quirino Lai

Copyright (C) 2019 Qingqin Hao et al. This is an open access article distributed under the Creative Commons Attribution License, which permits unrestricted use, distribution, and reproduction in any medium, provided the original work is properly cited.

\begin{abstract}
Emerging studies have reported circRNAs were dysregulated in HCC. However, the clinical value of these circRNAs remains to be clarified. Herein, we aimed to comprehensively explore their association with the diagnosis, prognosis, and clinicopathological characteristics of HCC. PubMed, EMBASE, Web of Science, and Cochrane Library databases were comprehensively searched for eligible studies up to October 30, 2018. The diagnostic effect was evaluated by the pooled sensitivity, specificity, and other indexes. The pooled hazard ratio (HR) for overall survival (OS) and recurrence free survival (RFS) was calculated to assess the prognostic value. Ten studies on diagnosis, 12 on prognosis, and 23 on clinicopathology were identified from the databases. A total of 11 upregulated and 11 downregulated circRNAs showed an association with clinicopathological features of HCC. For the diagnosis analyses, the pooled sensitivity, specificity, positive likelihood ratio (PLR), negative likelihood ratio (NLR), and diagnostic odds ratio (DOR) of circRNAs for HCC were 0.74 (95\%CI: 0.65-0.82) and 0.76 (95\%CI: 0.70-0.81), 3.1 (95\%CI: 2.5-3.8), 0.34 (95\%CI: 0.25-0.47), and 9 (95\%CI: 6-14), respectively. The area under SROC curve (AUC) was 0.81 (95\% CI: 0.78-0.84), indicating moderate diagnostic accuracy. In stratified analyses, the diagnostic performance of circRNAs varied based on the source of control and specimen type. For the prognosis analyses, increased expression of upregulated circRNAs was associated with worse OS (HR: 3.67 , 95\%: 2.07-6.48), while high expression of downregulated circRNAs was associated with better OS (HR: 0.38, 95\%: 0.30-0.48). In conclusion, this study reveals that circRNAs may serve as promising diagnostic and prognostic biomarkers for HCC. However, further investigations are still required to explore the clinical value of circRNAs.
\end{abstract}

\section{Introduction}

Hepatocellular carcinoma (HCC), a highly heterogeneous malignancy, is the second leading cause of cancer-related death worldwide $[1,2]$. Although major progress has been achieved in prevention, detection, diagnosis, and treatment, a total of 782000 cases diagnosed and 746000 deaths were estimated to occur in 2012 worldwide [3]. Currently, due to inefficient screening, HCC is often diagnosed at advanced stages; many patients therefore miss the optimal time for surgery $[4,5]$. Furthermore, failure to identify patients at high risk of metastasis and recurrence has also resulted in an unsatisfactory prognosis of HCC patients. Therefore, there is an urgent need for more effective biomarkers for early detection and prognosis prediction of HCC.
Circular RNAs (circRNAs), a novel class of noncoding RNA, are generated by 'backsplicing' of protein-coding mRNAs or linear noncoding RNA that join an upstream $3^{\prime}$ splice site and downstream $5^{\prime}$ splice site to form a covalently closed continuous loop [6]. They are highly stable, abundant and conserved, and involved in various physiological and pathological processes. However, the biological functions of most circRNAs are still unclear. Recently, emerging studies have revealed that aberrant circRNA expression has been observed in various cancers, such as colorectal cancer, breast cancer, gastric cancer, and HCC [7]. These circRNAs played crucial roles in the cancer-associated proliferation, angiogenesis, and metastasis and might be the key factors for cancer occurrence and development. To date, a series of articles have reported that circRNAs have great potential to serve as 
promising biomarkers for HCC. For instance, Qin et al. [8] found hsa_circ_0001649 was significantly downregulated in HCC. It might function in tumorigenesis and metastasis and could serve as a potential biomarker in the diagnosis of HCC $(\mathrm{AUC}=0.63)$. In addition, cSMARCA5 could inhibit the proliferation and migration of HCC cells. The downregulation of cSMARCA5 was significantly correlated with aggressive characteristics and might serve as an independent risk factor for overall survival (OS) and recurrence free survival (RFS) in HCC patients [9].

However, due to the variances in study design, sample size, patient characteristic, and detection methods, the clinical value of circRNAs for HCC has not yet been fully elucidated. Previously, four published meta-analyses have reported the diagnostic and prognostic value of circRNAs for human cancers [10-13]; however, they included relatively few studies and patients and did not perform detailed analyses to explore the diagnostic value of circRNAs for HCC. Therefore, we performed this systematic review and meta-analysis to explore the relationship between aberrant cirRNAs expression and the diagnosis, prognosis, and clinicopathological characteristics of HCC.

\section{Methods}

2.1. Search Strategy. This meta-analysis was conducted according to the PRISMA guideline (Supplement File S1) [14]. We comprehensively searched for the relevant articles in PubMed, EMBASE, Web of Science, and Cochrane Library databases (up to October 30, 2018) assessing the potential clinical utility of circRNAs for HCC. A combination of the Medical Subject Headings (MeSH) and title/abstract words was used: (liver neoplasia or carcinoma or neoplasm or cancer or tumor) and (circular RNAs or circRNAs). We also manually searched relevant reviews and bibliographies of eligible articles to find out other potential studies.

2.2. Eligibility Criteria. The eligible studies should meet the following criteria: (1) the diagnosis of HCC was pathologically confirmed; (2) about evaluating the relationship between circRNAs and the diagnosis, prognosis, or clinicopathological characteristics of HCC; (3) for diagnosis, studies could supply sufficient information to construct the diagnostic $2 \times 2$ tables; and (4) for prognosis, HR (hazard ratio) and its 95\% confidence interval (95\% CI) can be extracted or calculated from the studies [15]. The exclusion criteria were as follows: (1) duplicate articles; (2) case reports, letters, reviews, editorials, and meeting abstracts; and (3) insufficient data.

2.3. Data Extraction and Quality Assessment. The following data were extracted: first author, year of publication, country, sample size, clinicopathological features, circRNAs profiles, altered expression, specimen type, detection method, reference gene, diagnostic data, follow-up period, outcomes, and HRs with its 95\% CIs.

The quality of diagnostic studies was assessed with the Quality Assessment of Diagnostic Accuracy Studies 2 (QUADAS2). Meanwhile, the Newcastle-Ottawa Scale (NOS) was applied to assess the quality of prognostic studies [16], and a score $\geq 6$ indicates high quality.

All these processes were performed independently by two reviewers (HQQ and HYD). Any discrepancies were resolved by consensus.

2.4. Statistical Analysis. All analyses were performed with the RevMan5.3 (version 1.4), STATA 12.0 (STATA Corporation, College Station, TX), Meta-Disc 1.4, and Engauge Digitizer 4.1 software. HRs with $95 \%$ CI were directly extracted from each study, if provided, or calculated according to the methods clarified by Tierney et al. [15]. A bivariate meta-analysis model was employed to calculate the pooled sensitivity, specificity, likelihood ratio (LR), diagnostic odds ratio (DOR), and HR with 95\% CI, respectively. A summary receiver operator characteristic curve (SROC) was also established and corresponding AUCs with 95\% CI were calculated $[17,18]$. The Cochran-Q and Inconsistency index $\left(I^{2}\right)$ tests were applied to assess the heterogeneity among studies [19]. A $P$ value $(\leq 0.05)$ or $I^{2}$ value $(\geq 50 \%)$ indicated significant heterogeneity and the random-effects model was adopted. Otherwise, the fixed-effects model was used [20]. Spearman correlation coefficient was used to verify the threshold effect. To explore the sources of heterogeneity, we performed subgroup analysis and metaregression. Sensitivity analysis was further carried out to assess the robustness of the results. At last, publication bias was evaluated using Begg's funnel plot [21] and Deek's funnel plot, and $P>0.05$ indicated no potential publication bias. All tests were two-sided and $P<0.05$ was regarded as statistically significant.

\section{Results}

3.1. Literature Selection. As described in Figure 1, a total of 254 articles were initially identified and 151 studies remained after excluding duplicate studies. By screening the titles and abstracts, 116 articles were further excluded because of editorial, reviews, conference abstracts, or irrelevant research topic. As a result, 35 remaining articles were for full-text review, and then 6 papers were excluded due to conference abstracts or insufficient data. Ultimately, 29 articles were included in this study, including 23 studies [8, 9, 22-42] on clinicopathological features, 10 on diagnosis $[8,22,25-27,31-$ $33], 10$ on OS $[9,26,28,34,35,38-40,43,44]$, and 2 on RFS $[9,45]$.

3.2. Correlation of circRNAs Expression with Clinicopathological Features. A total of 22 circRNAs from 23 articles showed an association with clinicopathological features of HCC. As summarized in Table 1, hsa_circ_0128298, circRNA_100338, circHIPK3, Hsa_circ_001569, hsa_circ_0005075, circ-PVT1, circ-10720, circRNA101368, circ_001569, has_circ_0078710, and circ-ZEB1.33 were upregulated, whereas hsa_circ 0001445, circSMAD2, Hsa_circ_0001649, hsa_circ_0005986, CircC3P1, hsa_circ_0004018, cirZKSCAN1, cSMARCA5, hsa_circ_0003570, hsa_circ_0068669, and hsa_circ_0064428 were downregulated. Altered circRNAs expression was significantly associated with tumor stage, differentiation, size, numbers, vascular invasion, organ metastasis, and 


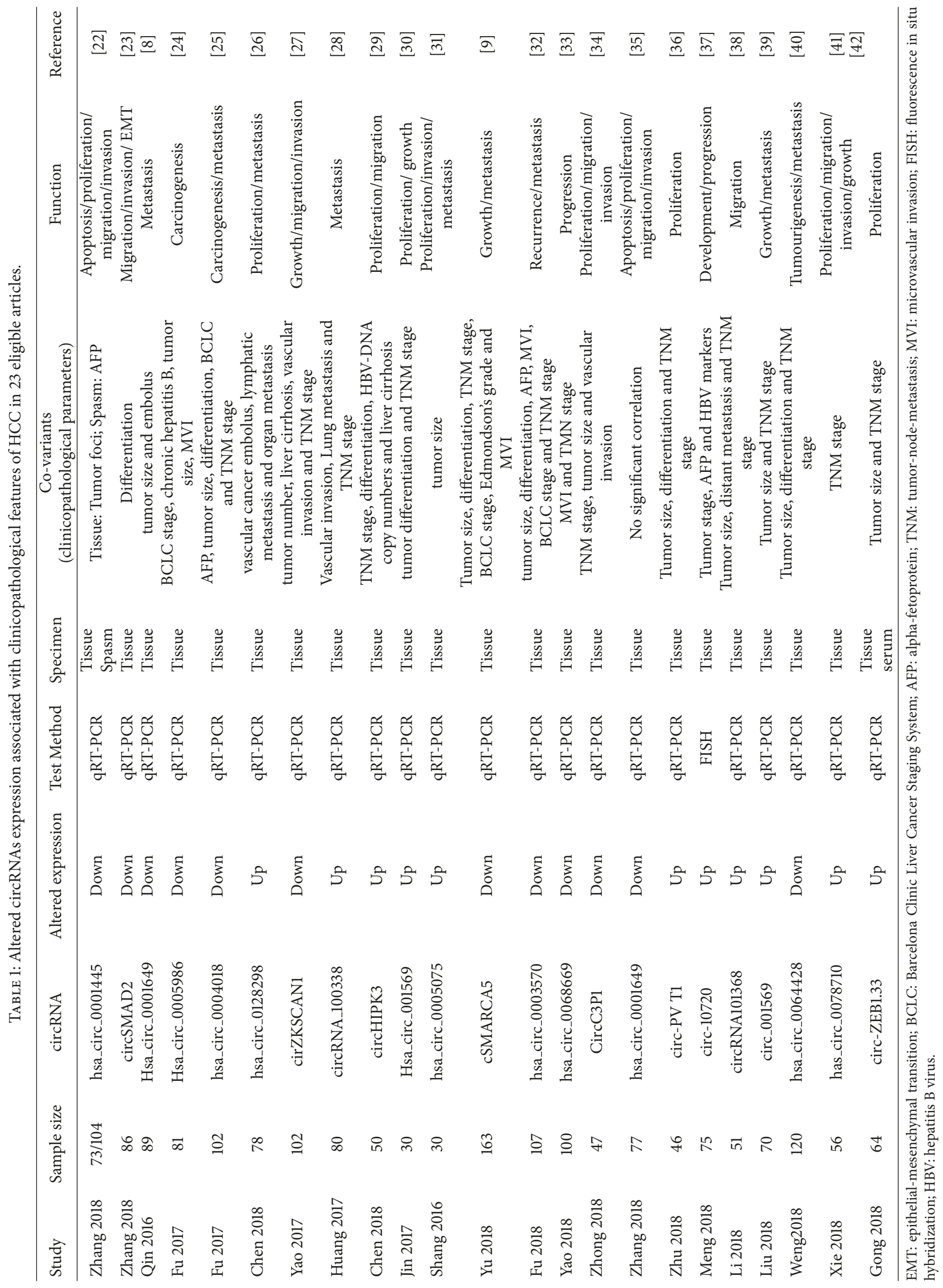




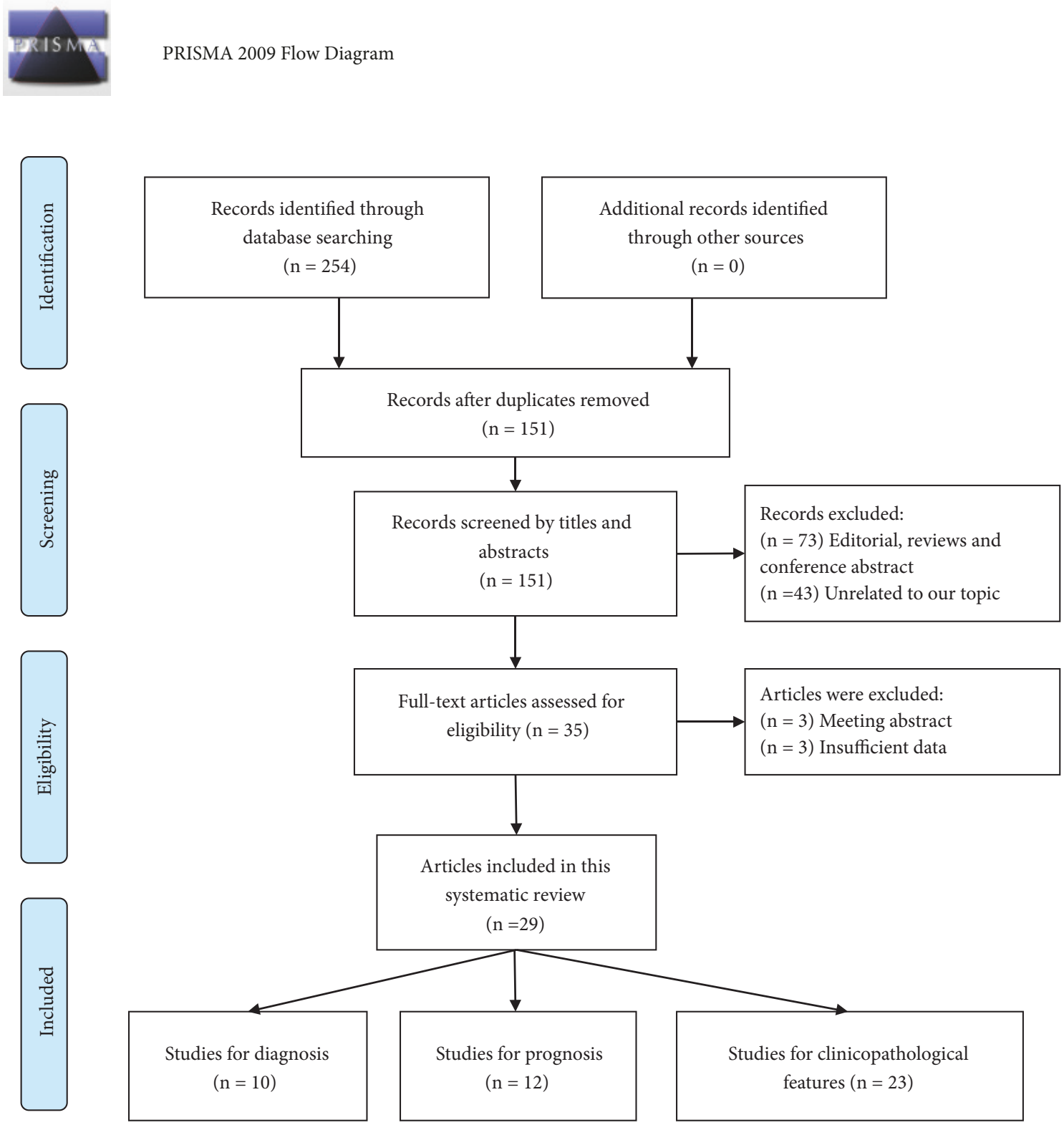

FIGURE 1: The flow diagram of the study selection process.

AFP (alpha-fetoprotein) in almost studies. They might play crucial roles in tumorigenesis and tumor progression of HCC. Additionally, some studies also showed a relationship of circRNAs expression with liver cirrhosis or chronic hepatitis B.

\section{Diagnostic Meta-Analysis}

4.1. Study Characteristics and Quality Assessment. The baseline characteristics of the eligible studies were summarized in Table 2. Ten studies from 8 articles with 712 cases and 811 controls were included. All of the studies were published from 2016 to 2018 and conducted in China. Most of patients were male and pathologically diagnosed with $\mathrm{HBV}$-associated HCC. The quantitative reverse transcription polymerase chain reaction (qRT-PCR) was used to measure the expression of 8 circRNAs, and the most common reference gene was GAPDH. In addition, specimens contain plasma and tissue. The quality of the studies was moderate. Further details of the quality assessment were summarized in Supplement Figure S1.

4.2. Pooled Diagnostic Performance. As shown in Figure 2, considerable heterogeneity was observed among these studies $\left(I^{2}=89.68 \%, P<0.01\right.$ for sensitivity; $I^{2}=75.64 \%, P<$ 0.01 for specificity). Therefore, a random-effect model was conducted. The pooled sensitivity, specificity, PLR, NLR, and DOR were 0.74 (95\%CI: 0.65-0.82), 0.76 (95\%CI: $0.70-$ 0.81 ), 3.1 (95\%CI: $2.50-3.80), 0.34$ (95\%CI: $0.25-0.47$ ), and 9 (95\%CI: 6 - 14), respectively. Moreover, the summary receiver 


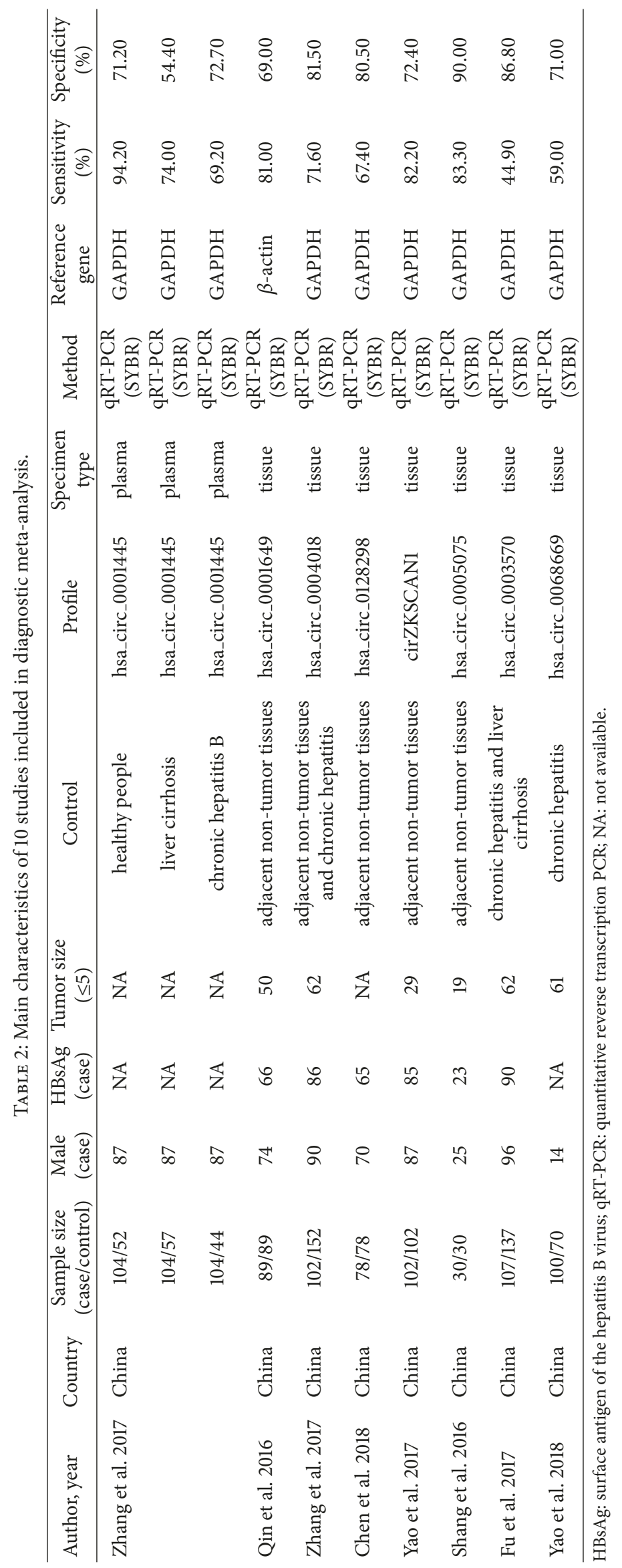



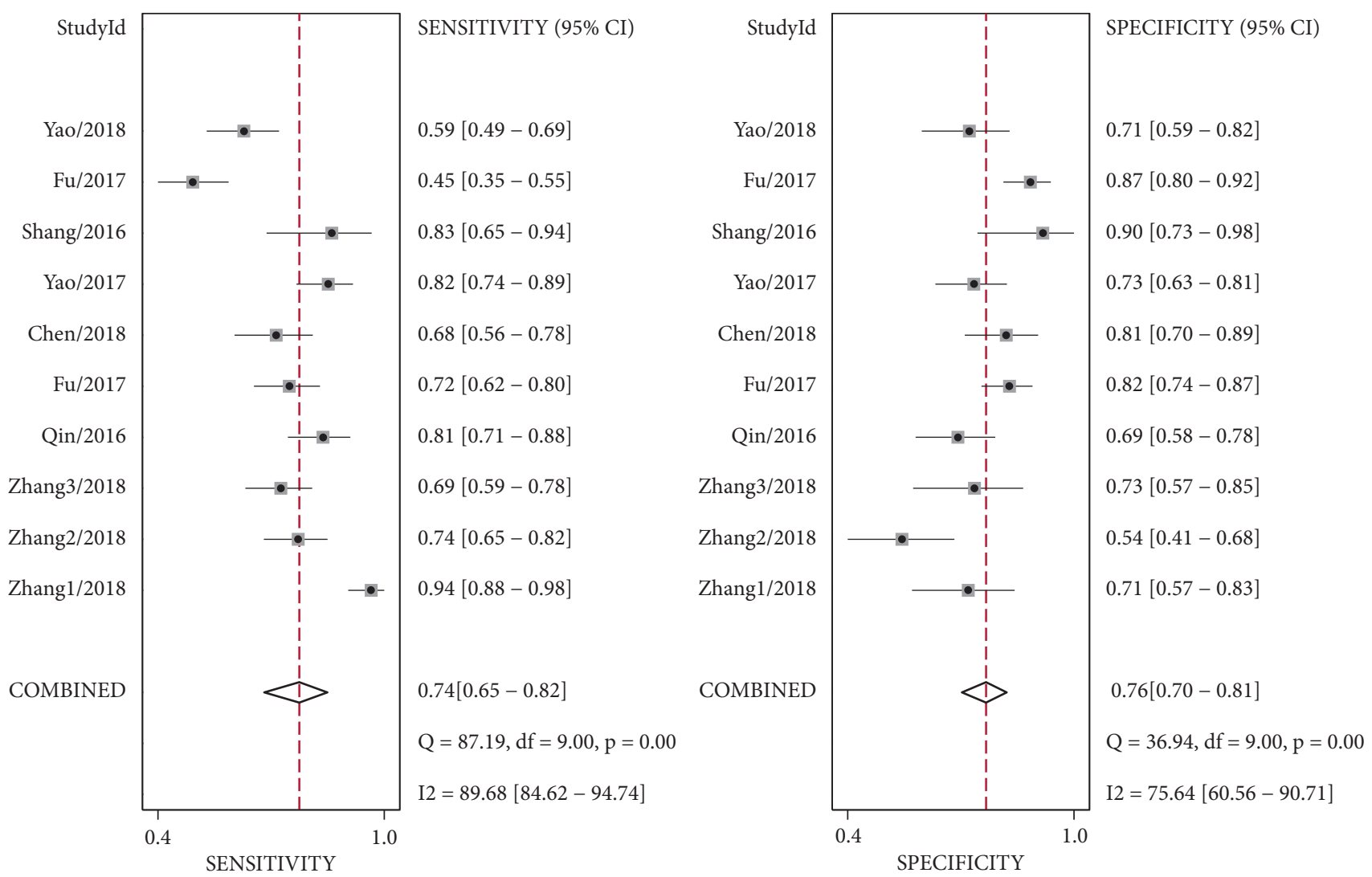

FIGURE 2: Forest plots of sensitivities and specificities of cirRNAs for the diagnosis of HCC.

operating characteristic curve (SROC) was also performed and the AUC was 0.81 (95\% CI: 0.78-0.84) (Figure 3), indicating circRNAs had potential diagnostic value for HCC. In this study, threshold effect, the important source of heterogeneity, was explored. The spearman correlation coefficient was 0.248 $(P=0.489)$, indicating no obvious threshold effect existed within included studies.

4.3. Subgroup Analysis and Meta-Regression Analysis. To explore the potential sources of heterogeneity, metaregression, and subgroup analyses were conducted according to the sample size, source of control, specimen type, reference gene, and male ratio. As presented in Table 3 , circRNAs could more efficiently discriminate HCC from healthy individuals or adjacent nontumor tissues than from benign diseases (sensitivity: 0.83 versus 0.64 , DOR: 14 versus 6 , and AUC: 0.81 versus 0.75 ), and the heterogeneity reduced significantly from $71.4 \%$ to $56.7 \%$ and $55.6 \%$, respectively. For the subgroup based on specimen type, plasma circRNAs might obtain a higher sensitivity and lower specificity ( 0.79 versus 0.68 and 0.65 versus 0.79 , respectively). In addition, compared with the overall results, there were no significant differences in the studies with male $(\geq 80 \%)$ or with GAPDH as a reference gene. According to the results of metaregression, none of these covariates above was responsible for the heterogeneity among included studies $(p>0.05)$. However, the source of control (RDOR: 2.65, 95\% CI: 0.94-7.46, $P=0.06$ ) might partially explain the heterogeneity.

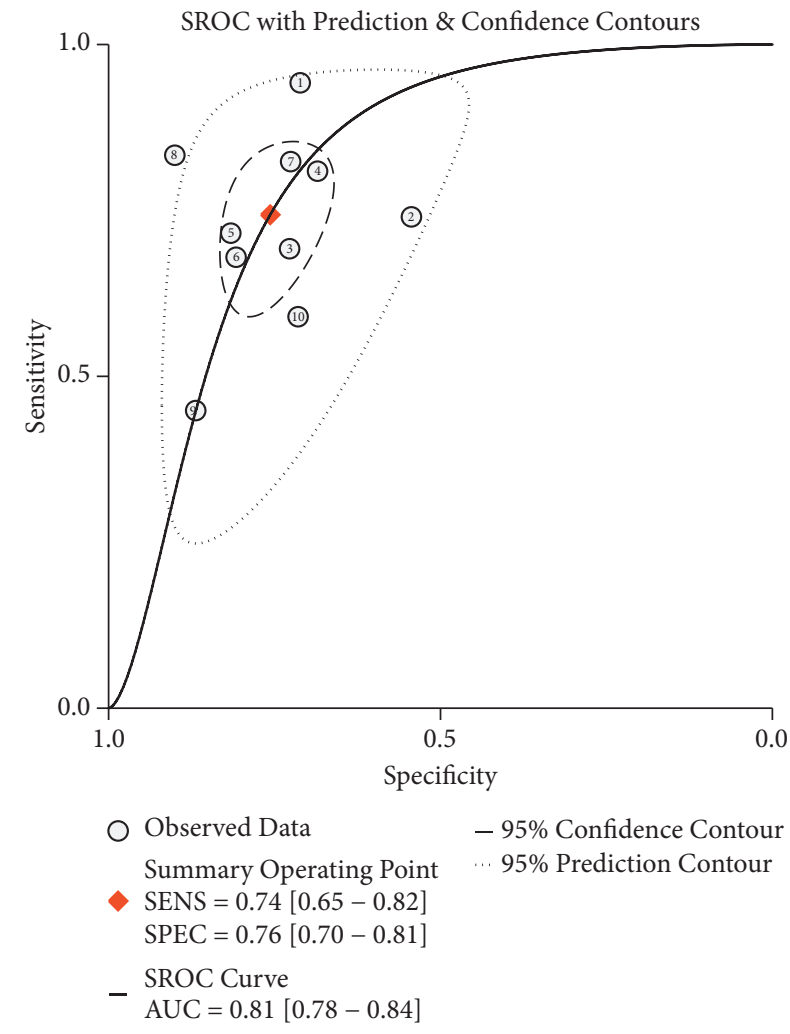

FIGURE 3: Summary receiver operating characteristic (SROC) curve of cirRNAs in the diagnosis of HCC. 


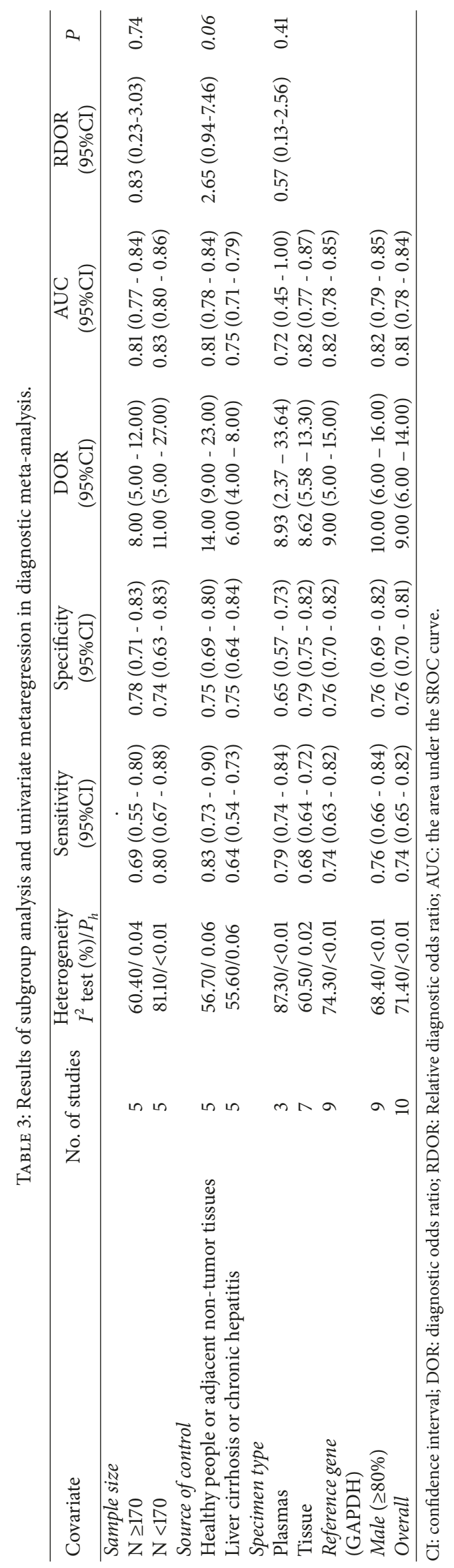




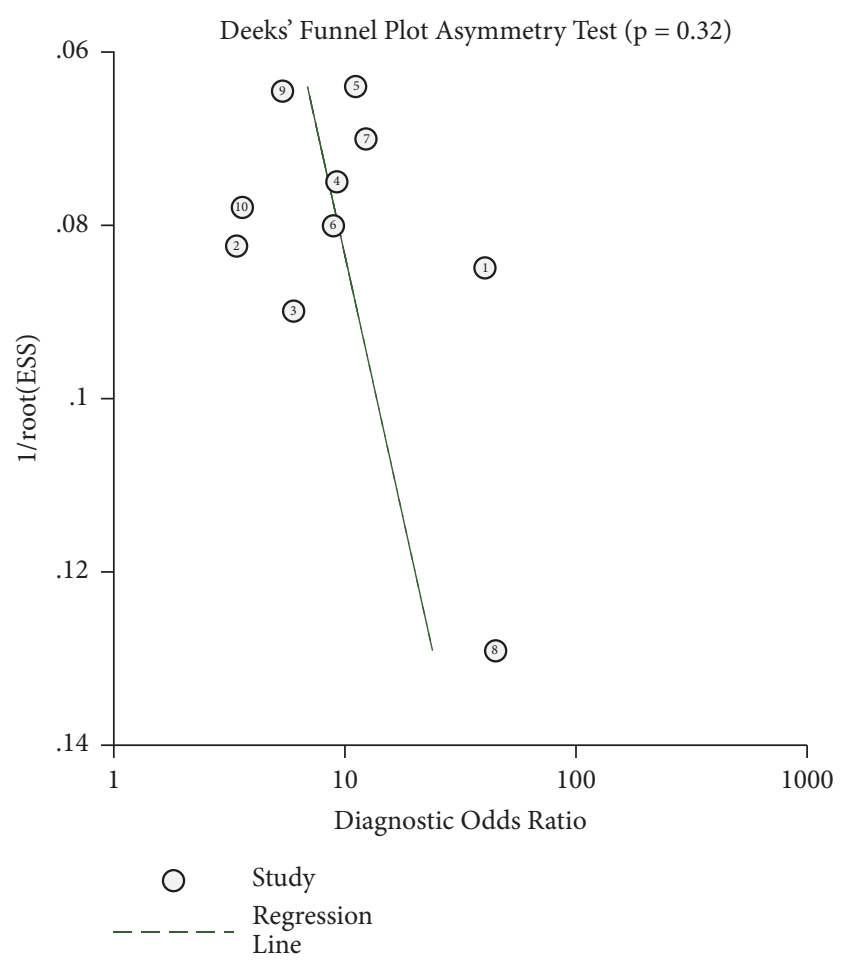

FIGURE 4: Deek's funnel plot to evaluate the publication bias of test accuracy.

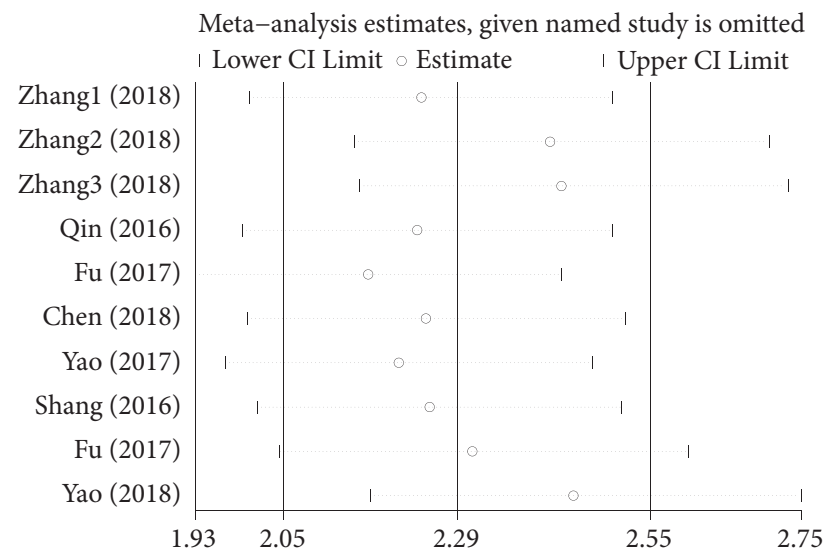

FIGURE 5: Sensitivity analysis of the overall pooled diagnostic studies (outlier detection analysis).

4.4. Publication Bias and Sensitivity Analysis. To evaluate the publication bias of the included studies, Deeks' funnel plot asymmetry test was performed. As indicated in Figure 4, a $P$ value of 0.32 suggested that there was no significant publication bias. Sensitivity analysis was further performed. As displayed in Figure 5, the results were stable and not significantly affected by any individual study.

\section{Prognostic Meta-Analysis}

5.1. Study Characteristics and Quality Assessment. As present in Table 4, 12 studies from 11 articles with 1185 cases were included in this prognosis analysis. All these studies were conducted in China and published from 2016 to 2018. The qRT-PCR and FISH were adopted to quantify the level of circRNAs in tissues with GAPDH and b-actin as reference genes. OS and RFS were used to evaluate the outcome of the cohorts. A total of 11 different circRNAs were investigated. Increased expression of hsa_circ_0128298, ciRS-7, circRNA101368, circ_001569, and circRNA_100338 and decreased expression of CircC3P1, cSMARCA5, hsa_circ_0001649, hsa_circ_0064428, circ-ITCH, and circMTO1 were associated with worse prognosis. HRs and 95\% CI were directly reported in 7 studies, and the remaining were extrapolated and calculated from Kaplan-Meier curves. The NOS scores varied from 5 to 7 , suggesting that the quality of included studies was moderate. Details of quality assessment were present in Supplement Table S1.

5.2. Association between circRNAs and Outcomes. Due to significant heterogeneity among studies existed $\left(I^{2}=92 \%, P\right.$ $<0.01$ ), a random-effects model was performed. As shown in Figure 6, the pooled HR of OS was 0.90 (95\%CI: 0.43-1.88) for high versus low cirRNAs expression. Stratified analysis according to altered expression was then performed. The pooled HR for upregulated circRNAs and downregulated circRNAs were 3.67 (2.07-6.48) and 0.38 (0.30-0.48), respectively (Figure 6(a)), and the heterogeneity reduced significantly from $92 \%$ to $47 \%$ and $0 \%$, respectively. The increased expression of upregulated circRNAs or decreased expression of downregulated circRNAs was significantly related to a worse prognosis. Metaregression analysis for this subgroup suggested that altered expression was the main source of heterogeneity $(P<0.01)$.

Additionally, two studies including 258 patients reported HRs for RFS. The overall result revealed that circRNAs expression was not associated with RFS in HCC patients (HR: 0.79, 95\%CI: 0.41-1.51, $P=0.47$ ) (Figure 6(b)).

5.3. Publication Bias. Publication bias was checked by Begg's funnel plot. As suggested in Figure 7, a $P$ value of 0.19 suggested that there was no significant publication bias among these studies.

\section{Discussion}

Increasing studies have demonstrated that circRNAs are relatively stable and detectable in body fluids and tissues and may serve as promising biomarkers for cancer diagnosis and prognosis [46]. Herein, we implemented this comprehensive review to evaluate the clinical value of circRNAs for HCC. According to the results of this study, the diagnostic accuracy of circRNAs for HCC was moderate. Moreover, altered circRNAs expression was significantly associated with tumor characteristic, and increased expression of upregulated circRNAs or decreased expression of downregulated circRNAs could predict worse OS in HCC patients. These findings indicate that circRNAs may serve as promising biomarkers for HCC diagnosis and prognosis prediction.

For diagnostic value, four previous meta-analyses concluded that the overall sensitivity, specificity, and NLR of 


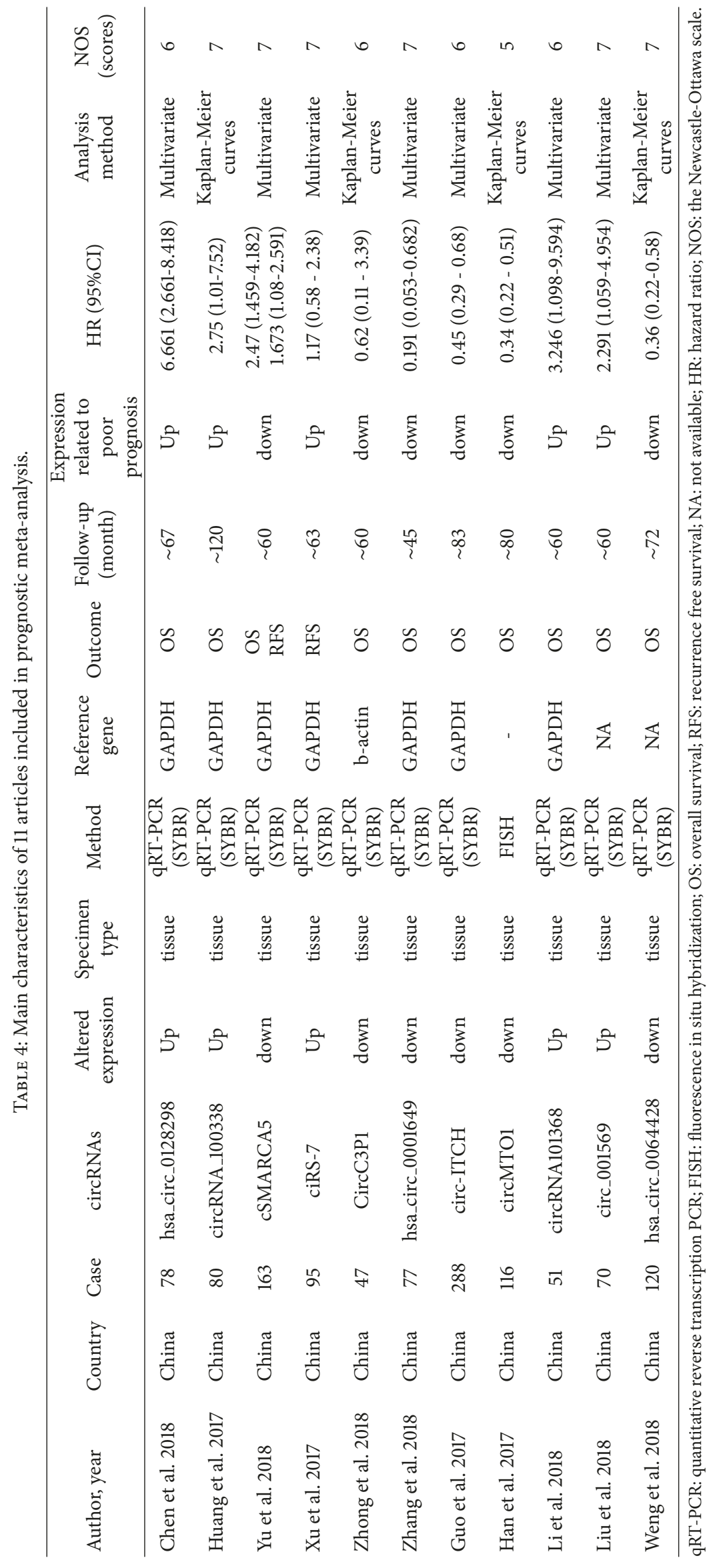




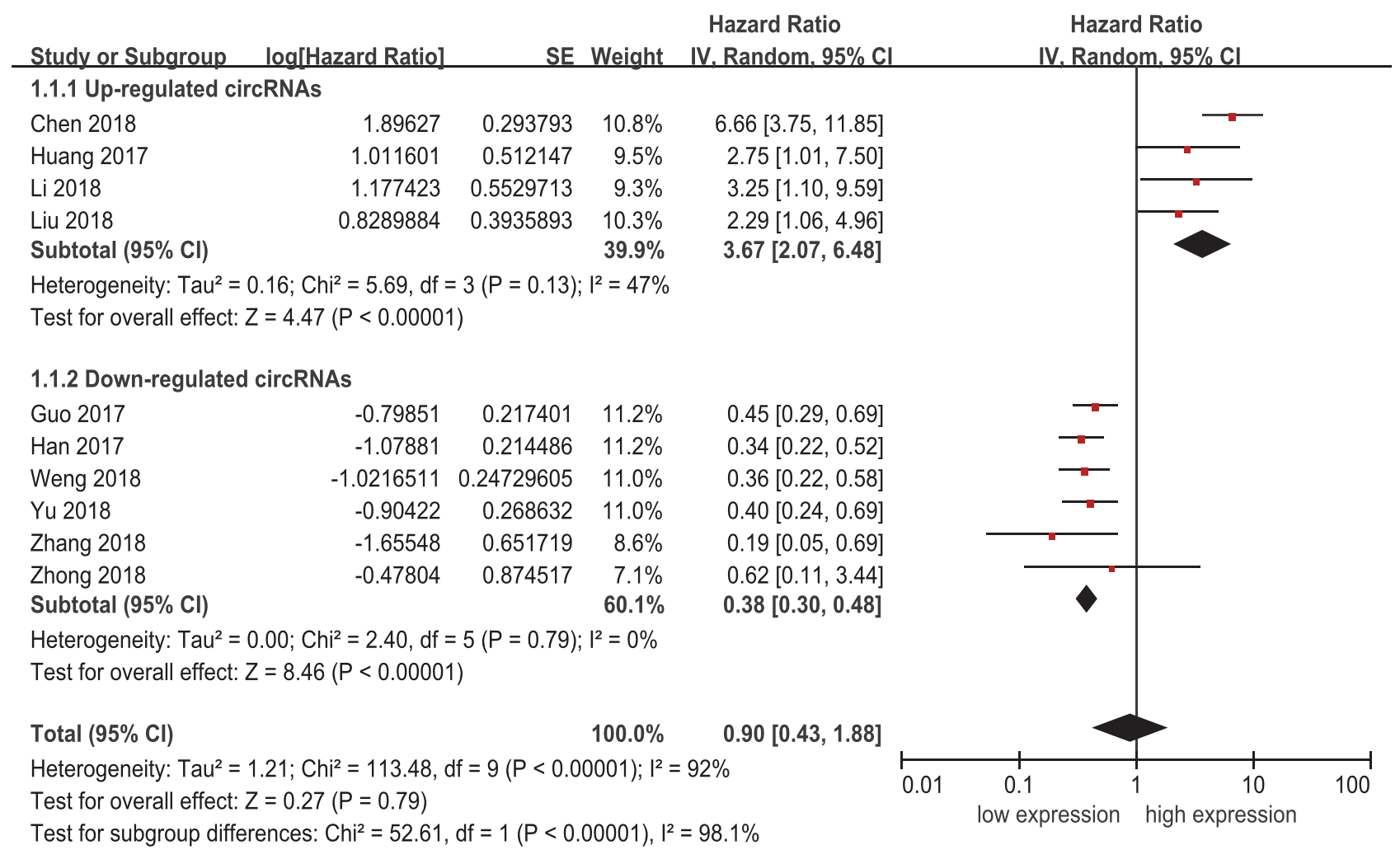

(a) OS

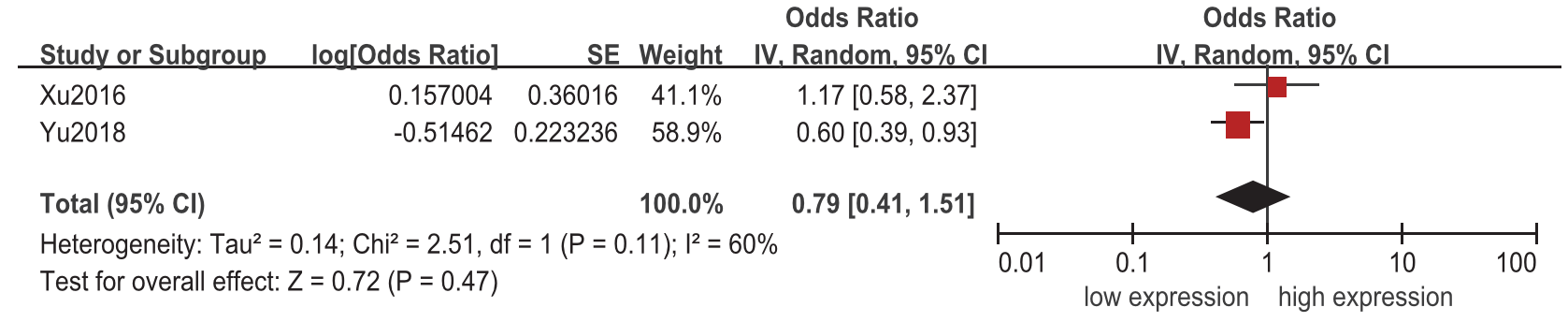

(b) RFS

FIGURE 6: Forest plot for the association between altered cirRNAs expression and survival in HCC. (a) Association with overall survival; (b) association with recurrence free survival. SE, standard error; IV, inverse variance methods; HR, hazard ratio; CI, confidence interval.

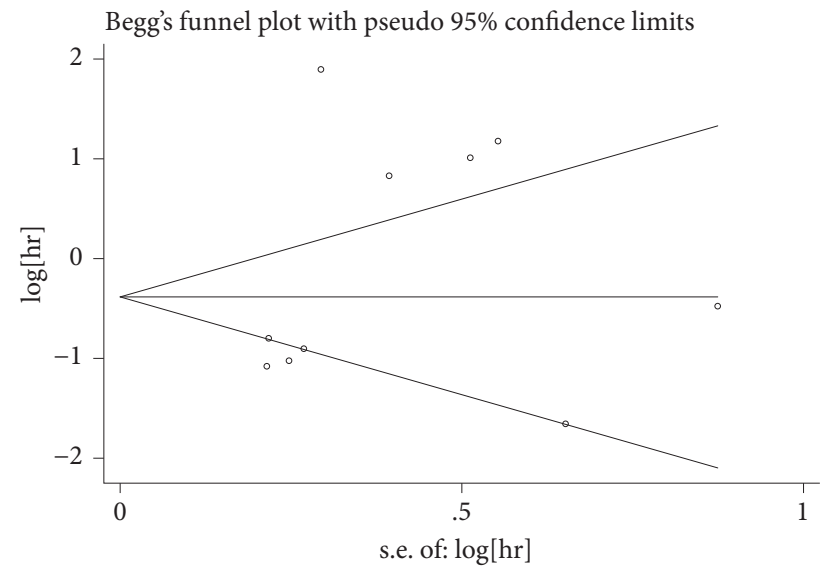

FIGURE 7: Begg's funnel plots for all of the included studies reported with overall survival.
circRNA for HCC were from 0.73 to $0.82,0.72$ to 0.79 , and 0.34, with PLR ranging from 3.40 to 3.51, DOR from 10.00 to 10.21 , and AUC from 0.83 to 0.86 , respectively [1013]. Consistent with these findings, in our study, the pooled sensitivity, specificity, and AUC of circRNAs were 0.74, 0.76 , and 0.81 , respectively, indicating a moderate diagnostic accuracy. The pooled DOR, a global measure of diagnostic performance [47], was 9, suggesting that circRNAs could effectively discriminate HCC patients from noncancerous controls. The pooled PLR was 3.1, suggesting that there was 3.1-fold higher possibility of altered expression of circRNAs for patients with HCC compared to those without. Likewise, NLR of 0.34 indicates that people with normal expression of circRNAs still have a 34\% chance of having HCC. In stratified analysis, expectedly, the diagnostic value of circRNAs varied according to the source of control. CircRNAs could more efficiently discriminate HCC from healthy individuals than from 
benign diseases. Furthermore, in accordance with previous studies [48-50], the characteristics of detection methods may also affect the diagnostic performance of circRNAs. Plasma circRNAs might obtain higher sensitivity and lower specificity in this study. Therefore, standardized protocol needs to be established to minimize protocol-based bias, and make the results more comparable.

Although the diagnostic performance of circRNAs was not good enough to confirm or exclude the diagnosis of HCC, circRNAs still have great advantages over the traditional clinical marker, and when combined with other biomarkers or clinical examinations, circRNAs may obtain a better diagnostic performance. As Fu et al. reported, hsa_circ_0004018 is a valuable biomarker for HCC diagnosis, with its superior sensitivity to alpha-fetoprotein (AFP) [25]. Similarly, Plasma hsa_circ_0001445 also had a higher diagnostic accuracy than AFP for distinguishing HCC patients from healthy people or patients with hepatitis B. And when combined, the efficiency in distinguishing HCC from healthy controls (AUC: 0.970, 95\% CI: 0.949-0.991), from cases of cirrhosis (AUC: 0.743 , 95\% CI: 0.664-0.821), or from cases of hepatitis B (AUC: 0.877, 95\% CI: 0.817-0.938) was higher [22]. However, further multicenter and high-quality studies are still required to explore their diagnostic value as promising biomarkers.

Advanced studies have revealed that plenty of circRNAs are differentially expressed in HCC. They play crucial roles in tumorigenesis and tumor progression $[6,7,51]$ and are significantly correlated with clinicopathological features, especially tumor characteristic. For instance, circSMAD2 inhibits the migration, invasion, and EMT of HCC cells by targeting miR629 [23] and markedly associates with the differentiation degree; circC3P1 acts as a tumor suppressor via enhancing PCK1 expression by sponging miR-4641 to inhibit HCC growth and metastasis. It negatively correlated with TNM stage, tumor size, and vascular invasion and might serve as a prognostic biomarker [34]. In addition, Hsa_circ_0005986 also functioned as microRNA sponge in tumorigenesis and accelerated cell proliferation by promoting the G0/ G1 to S phase transition in liver cancer cells and was correlated with tumor diameters, stage, and microvascular invasion [24]. All these evidences suggest that circRNAs may be risk factors for the outcome in HCC patients.

However, currently, it remains controversial whether circRNAs could serve as prognostic markers. Zhang et al. reported that hsa_circ_0001649 expression was a novel independent prognostic factor for a better OS of HCC patients [35], while upregulated circRNA_100338 closely correlated with a lower cumulative survival rate and metastatic progression in HCC patients with hepatitis B [28]. Moreover, elevated ciRS-7 expression in HCC showed a shorter time of tumor recurrence than that of patients with decreased ciRS-7 expression, but no statistical significance was observed [45]. In this meta-analysis, we found that upregulated or downregulated circRNAs were significantly associated with OS in HCC, and the predictive efficacy was significant, suggesting a value of employing circRNAs as biomarkers for HCC prognosis, which were consistent with previous meta-analysis and studies [13]. Regrettably, due to limited studies $(n=2)$, we failed to draw clear conclusions on the association between circRNAs and RFS in HCC patients. Therefore, further large-scale investigations are demanded to comprehensively and objectively investigate their promising prognostic value for HCC.

Compared with previous meta-analyses [10-13], we included more diagnostic studies, which would make our assessment more precise. What is more, further detailed analyses of diagnostic value of circRNAs for HCC were performed according to the sample size, source of control, specimen type, reference gene, and male ratio. In addition, the association between circRNAs expression and survival of HCC patients (OS and RFS) were also comprehensively evaluated. However, the following limitations merit consideration. Firstly, the number of studies and sample size are still relatively small, so our findings needed more large cohorts to validate. Secondly, due to unavailable original data, we failed to quantificationally evaluate the association of circRNAs with clinicopathological features and make more confirming conclusions. Thirdly, several HRs could not be directly extracted from 4 studies and were calculated from the Kaplan-Meier survival curves, which might be less reliable and biased our results. Fourthly, obvious heterogeneity existed across the included studies. The source of control and altered expression might be the sources of heterogeneity in diagnostic and prognostic meta-analysis, respectively. However, we failed to find other potential sources. Lastly, all included studies were conducted in China; therefore, our conclusions might not be universally suitable.

In summary, our meta-analysis indicates that aberrant circRNAs expression closely correlated with the clinicopathological characteristics of HCC, and it is possible that these circRNAs may serve as promising diagnostic and prognostic biomarkers for HCC. However, due to the limitations of this meta-analysis, well-designed, larger-size, and higher-quality prospective studies are required to confirm the clinical value of circRNAs as tumor markers and draw more definitive conclusions.

\author{
Abbreviations \\ circRNAs: Circular RNAs \\ HCC: Hepatocellular carcinoma \\ qRT-PCR: Quantitative reverse transcription PCR \\ DOR: Diagnostic odds ratio \\ LR: Likelihood ratio \\ AUC: $\quad$ Area under the SROC \\ SROC: $\quad$ Summary receiver operator characteristic \\ curve \\ QUADAS-2: Quality Assessment of Diagnostic \\ Accuracy Studies 2 \\ OS: Overall survival \\ RFS: $\quad$ Recurrence free survival \\ HR: $\quad$ Hazard ratio \\ NOS: $\quad$ Newcastle-Ottawa Scale.
}

\section{Ethical Approval}

This study does not include human participants or animals. 


\section{Conflicts of Interest}

The authors declare that they have no conflicts of interest.

\section{Acknowledgments}

This study is funded by Wuxi Science and Technology Department (no. WX18IIAN037).

\section{Supplementary Materials}

Supplementary 1. File S1. PRISMA 2009 Checklist (DOC).

Supplementary 2. Figure S1. Details of quality assessment by the QUADAS-2 tool in diagnosis meta-analysis. “-” in red, “?” in yellow, and “+” in green mean high risk, unclear risk, and low risk, respectively.

Supplementary 3. Table S1. Assessment of the risk of bias in each cohort study with the Newcastle-Ottawa Scale in prognosis meta-analysis (DOC).

\section{References}

[1] Y. Shimakawa, M. Lemoine, H. F. Njai et al., "Natural history of chronic HBV infection in West Africa: a longitudinal population-based study from the Gambia," Gut, vol. 65 , no. 12 , 2015.

[2] M. Maluccio and A. Covey, "Recent progress in understanding, diagnosing, and treating hepatocellular carcinoma," $C A$ : $A$ Cancer Journal for Clinicians, vol. 62, no. 6, pp. 394-399, 2012.

[3] A. Forner, M. Reig, and J. Bruix, "Hepatocellular carcinoma," The Lancet, vol. 391, no. 10127, pp. 1301-1314, 2018.

[4] M. Schwartz, S. Roayaie, and M. Konstadoulakis, "Strategies for the management of hepatocellular carcinoma," Nature Clinical Practice Oncology, vol. 4, no. 7, pp. 424-432, 2007.

[5] S. U. Ilikhan, M. Bilici, H. Sahin et al., "Assessment of the correlation between serum prolidase and alpha-fetoprotein levels in patients with hepatocellular carcinoma," World Journal of Gastroenterology, vol. 21, no. 22, pp. 6999-7007, 2015.

[6] C. Wong, F. H. Tsang, and I. O. Ng, "Non-coding RNAs in hepatocellular carcinoma: molecular functions and pathological implications," Nature Reviews Gastroenterology \& Hepatology, vol. 15, no. 3, pp. 137-151, 2018.

[7] L. Fu, Z. Jiang, T. Li, Y. Hu, and J. Guo, "Circular RNAs in hepatocellular carcinoma: functions and implications," Cancer Medicine, vol. 7, no. 7, pp. 3101-3109, 2018.

[8] M. Qin, G. Liu, X. Huo et al., "Hsa_circ_0001649: a circular RNA and potential novel biomarker for hepatocellular carcinoma," Cancer Biomarkers, vol. 16, no. 1, pp. 161-169, 2016.

[9] J. Yu, Q. Xu, Z. Wang et al., "Circular RNA cSMARCA5 inhibits growth and metastasis in hepatocellular carcinoma," Journal of Hepatology, vol. 68, no. 6, pp. 1214-1227, 2018.

[10] Z. Chen, L. Zhang, G. Han et al., "A meta-analysis of the diagnostic accuracy of circular RNAs in digestive system malignancy," Cellular Physiology and Biochemistry, vol. 45, no. 3, pp. 962-972, 2018.

[11] M. Wang, Y. Yang, J. Xu, W. Bai, X. Ren, and H. Wu, "CircRNAs as biomarkers of cancer: a meta-analysis," BMC Cancer, vol. 18, no. 1, 2018.
[12] H. Ding, Z. Lv, Y. Yuan, and Q. Xu, “The expression of circRNAs as a promising biomarker in the diagnosis and prognosis of human cancers: a systematic review and meta-analysis," Oncotarget, vol. 9, no. 14, 2018.

[13] X. Huang, W. Zhang, and Z. Shao, "Prognostic and diagnostic significance of circRNAs expression in hepatocellular carcinoma patients: A meta-analysis," Cancer Medicine, vol. 8, no. 3, pp. 1148-1156, 2019.

[14] D. Moher, A. Liberati, J. Tetzlaff, and D. G. Altman, "Preferred reporting items for systematic reviews and meta-analyses: the PRISMA statement," PLoS Medicine, vol. 6, no. 7, article no. e1000097, 2009.

[15] J. F. Tierney, L. A. Stewart, D. Ghersi, S. Burdett, and M. R. Sydes, "Practical methods for incorporating summary time-toevent data into meta-analysis," Trials, vol. 8, article 16, 2007.

[16] A. V. Margulis, M. Pladevall, N. Riera-Guardia et al., "Quality assessment of observational studies in a drug-safety systematic review, comparison of two tools: the Newcastle-Ottawa scale and the RTI item bank," Journal of Clinical Epidemiology, vol. 6, pp. 359-368, 2014.

[17] T. H. Hamza, L. R. Arends, H. C. Van Houwelingen, and T. Stijnen, "Multivariate random effects meta-analysis of diagnostic tests with multiple thresholds," BMC Medical Research Methodology, vol. 9, no. 1, article no. 73, 2009.

[18] J. B. Reitsma, A. S. Glas, A. W. S. Rutjes, R. J. P. M. Scholten, P. M. Bossuyt, and A. H. Zwinderman, "Bivariate analysis of sensitivity and specificity produces informative summary measures in diagnostic reviews," Journal of Clinical Epidemiology, vol. 58, no. 10, pp. 982-990, 2005.

[19] J. P. T. Higgins and S. G. Thompson, "Quantifying heterogeneity in a meta-analysis," Statistics in Medicine, vol. 21, no. 11, pp. 15391558, 2002.

[20] R. Dersimonian and N. Laird, "Meta-analysis in clinical trials," Controlled Clinical Trials, vol. 7, no. 3, pp. 177-188, 1986.

[21] C. B. Begg and M. Mazumdar, "Operating characteristics of a rank correlation test for publication bias," Biometrics, vol. 50, no. 4, pp. 1088-1101, 1994.

[22] X. Zhang, H. Zhou, W. Jing et al., "The circular RNA hsa_circ_0001445 regulates the proliferation and migration of hepatocellular carcinoma and may serve as a diagnostic biomarker," Disease Markers, vol. 2018, Article ID 3073467, 9 pages, 2018.

[23] X. Zhang, P. Luo, W. Jing, H. Zhou, C. Liang, and J. Tu, "circSMAD2 inhibits the epithelial-mesenchymal transition by targeting miR-629 in hepatocellular carcinoma," OncoTargets and Therapy, vol. 11, pp. 2853-2863, 2018.

[24] L. Fu, Q. Chen, T. Yao et al., "Hsa_circ_0005986 inhibits carcinogenesis by acting as a miR-129-5p sponge and is used as a novel biomarker for hepatocellular carcinoma," Oncotarget, vol. 8, no. 27, pp. 43878-43888, 2017.

[25] L. Fu, T. Yao, Q. Chen, X. Mo, Y. Hu, and J. Guo, "Screening differential circular RNA expression profiles reveals hsa_circ_0004018 is associated with hepatocellular carcinoma," Oncotarget, vol. 8, no. 35, pp. 58405-58416, 2017.

[26] D. Chen, C. Zhang, J. Lin, X. Song, and H. Wang, "Screening differential circular RNA expression profiles reveal that hsa_circ_0128298 is a biomarker in the diagnosis and prognosis of hepatocellular carcinoma," Cancer Management and Research, vol. Volume 10, pp. 1275-1283, 2018.

[27] Z. Yao, J. Luo, K. Hu et al., "ZKSCAN1 gene and its related circular RNA (circZKSCAN1) both inhibit hepatocellular carcinoma cell growth, migration, and invasion but through different 
signaling pathways," Molecular Oncology, vol. 11, no. 4, pp. 422437, 2017.

[28] X. Huang, Z. Huang, Y. Xu et al., "Comprehensive circular RNA profiling reveals the regulatory role of the circRNA100338/miR-141-3p pathway in hepatitis B-related hepatocellular carcinoma," Scientific Reports, vol. 7, no. 1, article no. 5428, 2017.

[29] G. Chen, Y. Shi, M. Liu, and J. Sun, “circHIPK3 regulates cell proliferation and migration by sponging miR-124 and regulating AQP3 expression in hepatocellular carcinoma," Cell Death \& Disease, vol. 9, no. 2, 2018.

[30] H. Jin, M. Fang, Z. Man, Y. Wang, and H. Liu, "Circular RNA 001569 acts as an oncogene and correlates with aggressive characteristics in hepatocellular carcinoma," International Journal of Clinical and Experimental Pathology, vol. 10, no. 3, pp. 29973005, 2017.

[31] X. Shang, G. Li, H. Liu et al., "Comprehensive circular RNA profiling reveals that hsa-circ-0005075, a new circular RNA biomarker, is involved in hepatocellular crcinoma development," Medicine, vol. 95, no. 22, 2016.

[32] L. Fu, S. Wu, T. Yao et al., "Decreased expression of hsa_circ_0003570 in hepatocellular carcinoma and its clinical significance," Journal of Clinical Laboratory Analysis, vol. 32, no. 2, 2018.

[33] T. Yao, Q. Chen, Z. Shao, Z. Song, L. Fu, and B. Xiao, "Circular RNA 0068669 as a new biomarker for hepatocellular carcinoma metastasis," Journal of Clinical Laboratory Analysis, vol. 32, no. 8, article no. e22572, 2018.

[34] L. Zhong, Y. Wang, Y. Cheng et al., "Circular RNA circC3P1 suppresses hepatocellular carcinoma growth and metastasis through miR-4641/PCK1 pathway," Biochemical and Biophysical Research Communications, vol. 499, no. 4, pp. 1044-1049, 2018.

[35] X. Zhang, S. Qiu, P. Luo et al., "Down-regulation of hsa_circ_0001649 in hepatocellular carcinoma predicts a poor prognosis," Cancer Biomarkers, vol. 22, no. 1, pp. 135-142, 2018.

[36] Y.-X. Zhu, H.-W. Fu, X. Lin et al., "Expression of hsa_circ_PVT1 in human hepatocellular carcinoma and its clinical significance," Medical Journal of Chinese People's Liberation Army, vol. 43, no. 3, pp. 211-216, 2018.

[37] J. Meng, S. Chen, J. Han et al., “Twistl regulates vimentin through Cul2 circular RNA to promote EMT in hepatocellular carcinoma," Cancer Research, vol. 78, no. 15, pp. 4150-4162, 2018.

[38] Z. Guan, J. Tan, W. Gao et al., "Circular RNA hsa_circ_0016788 regulates hepatocellular carcinoma tumorigenesis through miR-486/CDK4 pathway," Journal of Cellular Physiology, vol. 234, no. 1, pp. 500-508, 2018.

[39] H. Liu, L. Xue, C. Song, F. Liu, T. Jiang, and X. Yang, "Overexpression of circular RNA circ_001569 indicates poor prognosis in hepatocellular carcinoma and promotes cell growth and metastasis by sponging miR-411-5p and miR-432-5p," Biochemical and Biophysical Research Communications, vol. 503, no. 4, pp. 2659-2665, 2018.

[40] Q. Weng, M. Chen, M. Li et al., " Global microarray profiling identified $h s a_{-}$circ_0064428 as a potential immune-associated prognosis biomarker for hepatocellular carcinoma , Journal of Medical Genetics, vol. 56, no. 1, pp. 32-38, 2018.

[41] B. Xie, Z. Zhao, Q. Liu, X. Wang, Z. Ma, and H. Li, "CircRNA has_circ_0078710 acts as the sponge of microRNA-31 involved in hepatocellular carcinoma progression," Gene, vol. 683, pp. 253261, 2019.
[42] Y. Gong, J. Mao, D. Wu et al., "Circ-ZEB1.33 promotes the proliferation of human HCC by sponging miR-200a-3p and upregulating CDK6," Cancer Cell International, vol. 18, no. 1, 2018.

[43] D. Han, J. Li, H. Wang et al., "Circular RNA circMTO1 acts as the sponge of microRNA-9 to suppress hepatocellular carcinoma progression," Hepatology, vol. 66, no. 4, pp. 1151-1164, 2017.

[44] W. Guo, J. Zhang, D. Zhang et al., "Polymorphisms and expression pattern of circular RNA circ-ITCH contributes to the carcinogenesis of hepatocellular carcinoma," Oncotarget, vol. 8, no. 29, pp. 48169-48177, 2017.

[45] L. Xu, M. Zhang, X. Zheng, P. Yi, C. Lan, and M. Xu, "The circular RNA ciRS-7 (Cdrlas) acts as a risk factor of hepatic microvascular invasion in hepatocellular carcinoma," Journal of Cancer Research and Clinical Oncology, vol. 143, no. 1, pp. 17-27, 2017.

[46] H. Zhang, L. Jiang, D. Sun, J. Hou, and Z. Ji, "CircRNA: a novel type of biomarker for cancer," Breast Cancer, vol. 25, no. 1, pp. $1-7,2018$.

[47] A. S. Glas, J. G. Lijmer, M. H. Prins, G. J. Bonsel, and P. M. M. Bossuyt, "The diagnostic odds ratio: a single indicator of test performance," Journal of Clinical Epidemiology, vol. 56, no. 11, pp. 1129-1135, 2003.

[48] L. Dong, P. Qi, M.-D. Xu et al., "Circulating CUDR, LSINCT-5 and PTENP1 long noncoding RNAs in sera distinguish patients with gastric cancer from healthy controls," International Journal of Cancer, vol. 137, no. 5, pp. 1128-1135, 2015.

[49] A. Baraniskin, J. Kuhnhenn, U. Schlegel et al., "Identification of microRNAs in the cerebrospinal fluid as marker for primary diffuse large B-cell lymphoma of the central nervous system," Blood, vol. 117, no. 11, pp. 3140-3146, 2011.

[50] Q. Q. Hao, G. Y. Chen, J. H. Zhang, J. Sheng, and Y. Gao, "Diagnostic value of long noncoding RNAs for hepatocellular carcinoma," Medicine, vol. 96, no. 28, article no. e7496, 2017.

[51] L. Qiu, Y. Wu, X. Yu, Q. Tang, L. Chen, and K. Chen, "The emerging role of circular RNAs in hepatocellular carcinoma," Journal of Cancer, vol. 9, no. 9, pp. 1548-1559, 2018. 


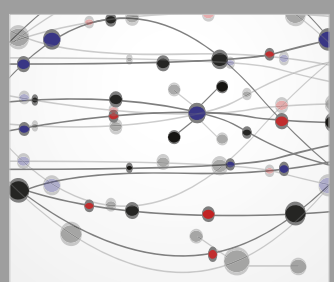

The Scientific World Journal
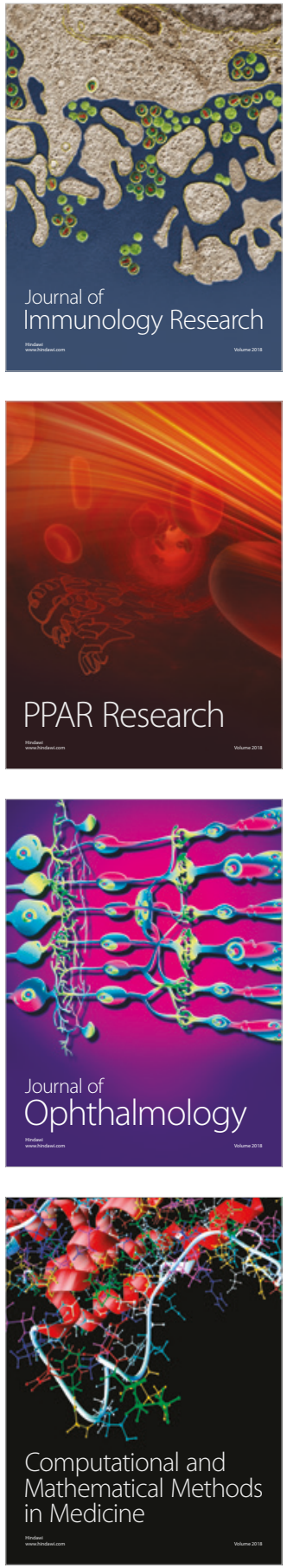

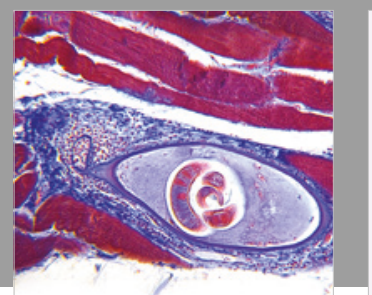

Gastroenterology Research and Practice

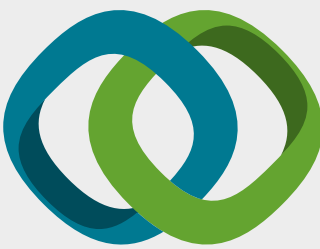

\section{Hindawi}

Submit your manuscripts at

www.hindawi.com
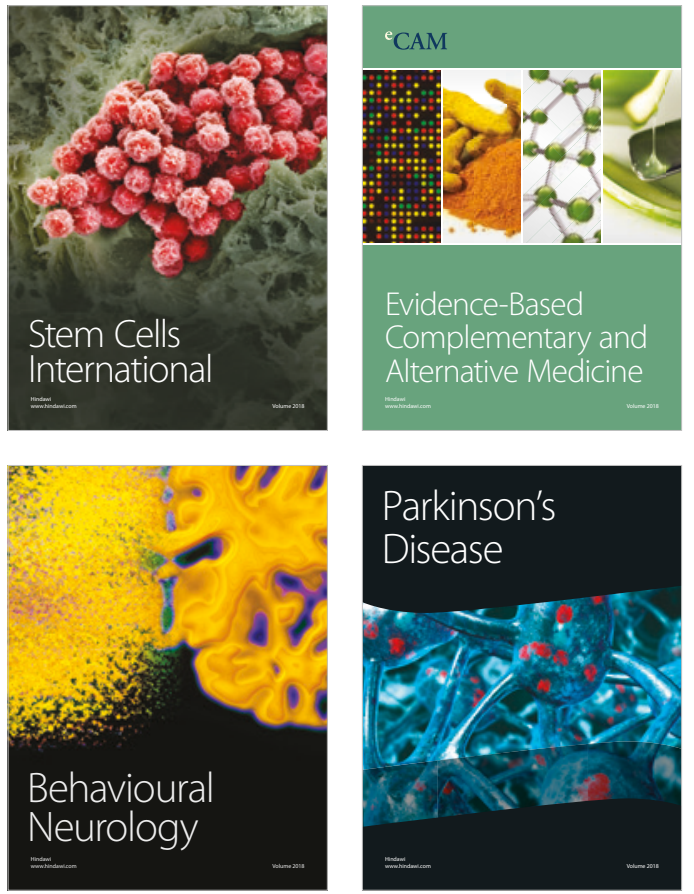

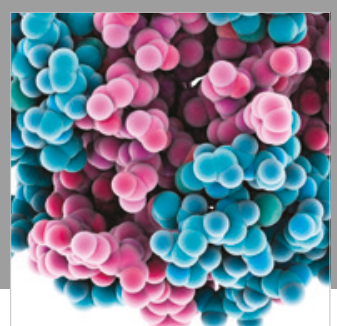

ournal of

Diabetes Research

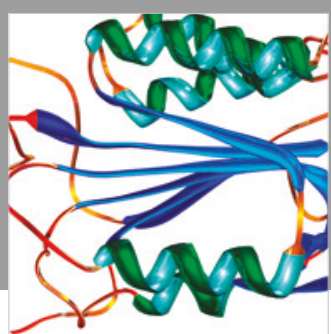

Disease Markers
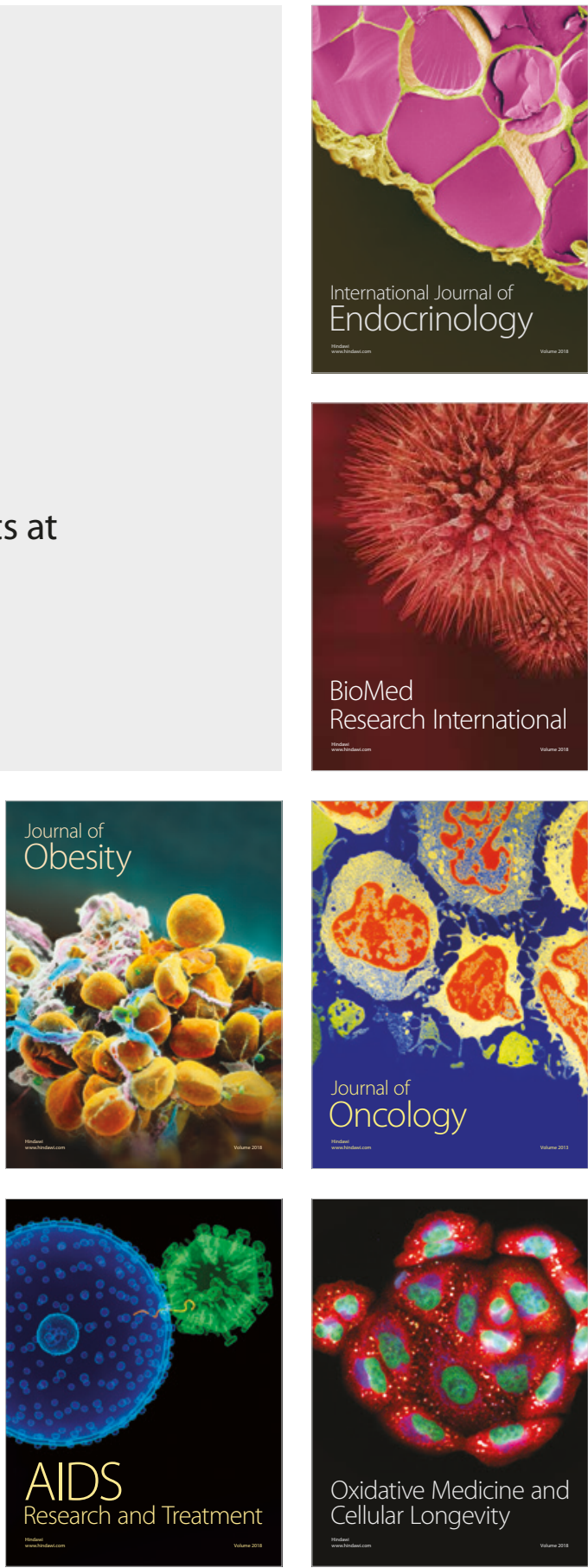